\title{
Reflections of a Clinician on the Current Trends in Clinical Neuroscience - Molecular Neurobiology and/or Connectome?
}

\author{
Leontino Battistin
}

Department of Neurosciences, University of Padova Medical School, Padova, Italy and IRCCS San Camillo Neurorehabilitation Research Hospital, Venice, Italy

1 n clinical neuroscience, the search to discover the cause and therapy for diseases was previously performed through the integrated approach of clinical observations with molecular neurobiology and neuroimaging. The new approach called "connectome" is mostly used in neuropsychological and behavioural studies correlated with neuroimaging data. However, to discover the causes and therapy of a disease, we should study not only connectivity but also cell processes and intercellular communications. Future research should be linked with clinical molecular neurobiology, and thus become true translational research.

\section{Keywords}

Clinical observation, connectome, molecular neurobiology, neuroimaging, Parkinson's disease, translational research

Disclosure: Leontino Battistin is a member of the journal's editorial board; he has nothing further to disclose in relation to this article.

Review Process: This article is a short opinion piece and has not been submitted to external peer reviewers; it was reviewed by another member of the editorial board before publication.

Authorship: The named authors meets the criteria of the International Committee of Medical Journal Editors for authorship for this manuscript, takes responsibility for the integrity of the work as a whole and has given final approval for the version to be published.

open Access: This article is published under the Creative commons Attribution Noncommercial License, which permits any non-commercial use, distribution, adaptation and reproduction provided the original author and source are given appropriate credit. (C) The Author 2018.

Received: 25 April 2018

Published Online: 6 June 2018

Citation: European Neurological Review. 2018;13(1):16-7

Corresponding Author: Leontino Battistin,

Department of Neurosciences, University of

Padova Medical School, Via Giustiniani 5, 35128

Padova, Italy. E: leontino.battistin@unipd.it

Support: No funding was received in

the publication of this article.
The main questions for a neurologist at the present time are: what are the fundamental lanes of research in clinical neuroscience and what are the ultimate goals of our investigations? Since the primary mission of a clinician is to be of help to patients, a clinical investigator has to keep searching to discover the aetiology and pathology of diseases in order to be able to reach the best diagnostic and preventive procedures, as well as the most efficacious therapies and rehabilitation actions that should be tailored for each patient. So, bearing this in mind as a clinical neuroscientist, we should reflect on the current state of the art in our field of research. In my opinion, a brief historical review of the evolution of knowledge in Parkinson's disease (PD) is the best way to debate the evolution of studies in clinical neuroscience.

In the 1950s, neuroleptic drugs (chlorpromazine, reserpine, etc.) were thought efficacious in the therapy of psychoses where patients showed the appearance of extrapyramidal symptoms as side effects. In 1957, Arvid Carlsson showed the deficit of dopamine in the brain of reserpinised rats with extrapyramidal signs; treating the rats with levodopa (L-dopa) was successful and the extrapyramidal signs disappeared. ${ }^{.}$In the 1960s and 1970s, L-dopa therapy became the "miracle" for thousands and thousands of patients with PD throughout the world.

In the 1980s, dopamine agonists added further strength to dopaminergic therapy of PD and also triggered new biochemical investigations in neurodegenerative diseases. Thus, in the 1980s it was shown that some patients with PD had cognitive impairments and that the use of anticholinergic drugs - used before L-dopa - was very negative for cognitive performance. On the basis of such observations, the first trial of cholinergic stimulation of dementia started with the use of anticholinesterase inhibitors.

In the 1990s a great deal of attention was paid to non-motor symptoms and especially to the ones that are detectable many years before PD diagnosis. These pre-clinical symptoms became of paramount importance for prevention. Also at this time, a lot of attention was paid to patients' quality of life, as well as to all rehabilitation techniques, both motor and cognitive. As far as prevention is concerned, eating a healthy diet and practising physical activity have been demonstrated to be key elements for an anti-PD lifestyle. ,.3 $^{2}$ Furthermore, in the 1990s there was an impressive growth of research in the neuroimaging field, providing quite important data which proved useful for diagnostic procedures and allowing a deeper knowledge in the physiopathology of PD and related disorders.

The integrated approach of research in PD was, on the one hand, the clinical observation of patients, and on the other molecular neurobiological study - that is, chemistry, physiology, immunology, pharmacology, etc. - both in animal models and in patients, combined with neuroimaging research.

Currently, a new approach is growing, the so-called "network connectivity" - that is, the research on neural connections and their significance in the function of the brain. This approach is certainly a positive one and it is very much used by investigators in neuropsychological and behavioural 
studies; thus, in this field there was a kind of explosion during the last 2-3 decades in correlating neuropsychological findings with neuroimaging data. ${ }^{4}$ It has also been quite frequently reported on by the media, fascinated by the many colour images of the working brain.

This network connectivity has certainly added new knowledge and provided a better understanding of the physiology of the brain, particularly in the cognitive and behavioural fields. Furthermore, such an approach could be useful in investigating the quite complex processes involved in neurorehabilitation. We are now at a point where an approach such as so-called "connectomics", related to neuroimaging, could add new information and data on the causes of brain diseases and their therapies.

Within the organisation of the nervous system, the cell and its environment can be considered the "centre", and the fibres are the "vehicles" of the messages given by the cells. If we want to discover the causes of a disease, we have to study cell processes, especially at the membrane level, and what is going wrong in such processes at the different levels of the brain morpho-functional organisation. In other words, we should look to the centre of the problem-that is, the cell. Obviously, if the cell is affected by a pathological process, that determines an impairment in the electrochemical processes, such altered processes can be propagated through intercellular communication channels to other sites.

From this point of view it is not clear, at least to me, how investigations based only on neuroimaging data, to obtain the so-called "network connectivity" could give complete and relevant information on the multi-faceted processes that cause cellular pathology and consequently neurological disease. Therefore, I have serious doubts that an approach relying solely on "connectome-imaging" could give definitive answers to help discover the causes and potential therapies of diseases. This fact makes the difference with the method of integrating clinical neurobiological techniques with neuroimaging that has been used in studying PD and related diseases. This approach has, as we have seen, been very beneficial for patients and could be defined as translational research, whereas the connectome method seems mostly to provide basic research, at least up to now. However, I must say that the connectome program has certainly been useful for neuroimaging research, as well as for the support to research given by public and private institutions.

I agree with Agnati and Fuxe ${ }^{5}$ that organisation of the nervous system is like a big tree with many branches and that electro-chemical processes at the cellular level, neuron and glia cells, the fibres and interstitial fluid modes of intercellular communication, should be considered interrelated branches to be investigated according to an integrated approach. In this context, the connectome program could certainly be useful, but it should not simply be considered as imaging research.

The above-mentioned reflections are in line with the evaluations of Raichle ${ }^{6}$ and of Agnati et al. ${ }^{7}$ with the "hyper-network" connectivity; both authors emphasised the need to base future research in this field on the link with cellular and molecular neurobiology. I am confident that this method of translational research will be the real future.

So, turning to the aim of this article, I should now give an answer to the question "and/or" in the title. My answer is "and" - that is, that the connectome-neuroimaging program should be strictly linked to clinical molecular neurobiology.

In the 1980s, there were great hopes that introducing spectroscopy research with magnetic resonance would have been a breaking point to start neurochemical research in humans; this did not happen - the industries preferred to continue mostly with the neuroimaging approach and therefore they focused on neuropsychological studies, and a great number of neuroscientists followed for various reasons. I hope that scientific societies and especially neurological societies will promote debate and scientific discussion on the above-mentioned points. $\square$
1. Carlsson $A$, Lindqvist $M$, Magnusson T. 3,4-dihydroxyphenylalanine and 5-hydroxytryptophan as reserpine antagonists. Nature. 1957;180:1200.

2. Bracco F, Malesani R, Saladini M, Battistin L. Protein redistribution diet and antiparkinsonian response to levodopa. Eur Neurol. 1991;31:68-71.

3. Dam M, Tonin P, Casson S, et al. Effect of conventional and sensory-enhanced physiotherapy on disability of
Parkinson's disease patients. Adv Neurol. 1996;69: $551-5$

4. Sporns $\mathrm{O}$. The human connectome: a complex network. Ann N Y Acad Sci. 2011;1224:109-25.

5. Agnati LF, Fuxe K. New concepts on the structure of the neuronal networks: the miniaturization and hierarchical organization of the central nervous system. Biosci Rep. 1984;4:93-8.
6. Raichle ME. The restless brain: how intrinsic activity organize brain function. Philos Trans R Soc Lond B Biol Sci. 2015;370: 20140172.

7. Agnati LF, Marcoli M, Maura G, et al. The brain as a "hyper-network": the key role of neural networks as main producers of the integrated brain actions especially via the "broadcasted" neuroconnectomics. J Neural Transm. 2018;125:883-97. 